\title{
Sensibilidade a Herbicidas de Acessos de Aguapé Coletados em Reservatórios do Estado de SÃo PaUlo ${ }^{1}$
}

\author{
Chemical Control of Different Water Hyacinth Accesses
}

CARDOSO L.R. ${ }^{2}$, MARTINS D. ${ }^{3}$ e TERRA, M.A. ${ }^{4}$

\begin{abstract}
RESUMO - Com o objetivo de avaliar o efeito de herbicidas em diferentes acessos de aguapé coletados em reservatórios de hidrelétricas do Estado de São Paulo, foi realizado um estudo no Núcleo de Pesquisas Avançadas em Matologia da FCA-UNESP, campus de Botucatu. A escolha das plantas geneticamente diferentes foi feita com base em estudos de variabilidade genética, nos quais se utilizou a técnica de RAPD. Avaliou-se o efeito dos herbicidas imazapyr nas doses de 62,5 e 125,0 g e.a. ha ${ }^{-1}$, glyphosate a 1.680 e 3.360 g e.a. ha ${ }^{-1}+0,5 \%$ V/V de Extravon, diquat a 480 e 960 g i.a. ha ${ }^{-1}$ e 2,4-D a 670 e 1.340 g e.a. ha-1. Os seis acessos escolhidos foram colocados em caixas plásticas de $28,0 \times 14,0 \times 12,0 \mathrm{~cm}$, contendo 4 litros de água. A aplicação dos produtos foi realizada com um simulador de pulverização pressurizado com ar comprimido, equipado com barra de aplicação com quatro bicos de jato plano Teejet 110.02 VS. A pressão constante de trabalho foi de 1,6 bar, e o consumo de calda, de $193 \mathrm{~L} \mathrm{ha}^{-1}$. A velocidade de aplicação foi de $3,69 \mathrm{~km} \mathrm{~h}^{-1}$. Durante as aplicações, a temperatura do ar foi de $25{ }^{\circ} \mathrm{C}$ e a umidade relativa de $73 \%$. Foram realizadas avaliações visuais de controle aos 3 , $5,7,11,21$ e 28 dias, nas quais 0 consistiu em nenhum controle e 100 em morte de plantas. Todos os herbicidas e doses testados proporcionaram controle eficiente das plantas de aguapé, e os seis acessos estudados responderam de forma semelhante.
\end{abstract}

Palavras-chave: 2,4-D, glyphosate, imazapyr, diquat, Eichhornia crassipes.

\begin{abstract}
A study was carried out at the Advanced Weed Science Research Nucleus , UNESP/ Botucatu, to verify the effect of herbicides on different collected accesses of water hyacinth in hydroelectric reservoirs in São Paulo. The different genetic materials were chosen based on studies of genetic variability, using the RAPD technique. The herbicides and rates evaluated were imazapyr at 62.5 and $125.0 \mathrm{~g}$ a.e. $\mathrm{ha}^{-1}$, glyphosate at 480 and $960 \mathrm{~g}$ a.e. ha $\mathrm{h}^{-1}$, diquat at 1.680 and 3.360g a.i. ha $+5 \% \mathrm{~V} / \mathrm{V}$ of Extravon and 2.4-D at 670 and $1.340 \mathrm{~g}$ a.i. ha ${ }^{1}$. Six chosen accesses were placed in a box of $28.0 \times 14.0 \times 12.0 \mathrm{~cm}$ containing $4 \mathrm{~L}$ water. Herbicide was applied by using a spray simulator pressurized with compressed air, with four "Teejet" $110.02 \mathrm{VS}$ flat fan nozzles. The constant work pressure was $1.6 \mathrm{bars}$, and the solution volume, $193 \mathrm{~L} \mathrm{ha}^{-1}$. Application speed was $3.69 \mathrm{~km} / \mathrm{h}$. During herbicide application, the air temperature was $25^{\circ} \mathrm{C}$ and the relative humidity, $73 \%$. Visual control evaluations were carried out on the $3^{\text {rd }}$, $5^{\text {th }}, 7^{\text {th }}, 11^{\text {th }}, 21^{\text {th }}$ and $28^{\text {th }}$ days after herbicide application. All the tested herbicides and rates provided an efficient control of water hyacinth plants, with the six accesses showing similar responses to the herbicide treatments.
\end{abstract}

Key words: 2,4-D, glyphosate, imazapyr, diquat, Eichhornia crassipes.

1 Recebido para publicação em 10.9.2002 e na forma revisada em 20.10.2002.

Parte da dissertação do primeiro autor apresentada para obtenção do título de Mestre.

2 Eng.-Agr., M.S., Faculdade de Ciências Agronômicas da Universidade Estadual Paulista - FCA/UNESP, Cx. Postal 237, 18603-970 Botucatu-SP; ${ }^{3}$ Professor Dr., Departamento de Produção Vegetal, FCA/UNESP; ${ }^{4}$ Eng.-Agr., FCA/UNESP. 


\section{INTRODUÇÃO}

Nos Estados Unidos, em especial nos estados da Flórida, Texas, Louisiana e Califórnia, aproximadamente 6 milhões de dólares foram gastos com herbicidas no controle de aguapé (Eichhornia crassipes) (FAO, 1995). Macrófitas aquáticas em desequilíbrio nos corpos hídricos apresentam taxa de crescimento elevada e produzem grandes quantidades de biomassa. De acordo com Bitar \& Sikar (1990), no reservatório de Salto Grande-SP, o aguapé apresentou crescimento na taxa de $30,93 \mathrm{~g}$ ao dia por indivíduo. A ocupação da superfície da água gera inconvenientes socioeconômicos que limitam o método de manejo a ser utilizado na área infestada.

Proporcionalmente aos recursos hídricos do Brasil, são poucos os ambientes eutrofizados que apresentam sérios problemas com plantas aquáticas. Os exemplos mais flagrantes localizam-se nas proximidades de grandes centros urbanos, como São Paulo, Belo Horizonte, Rio de Janeiro e Recife. Nestes ambientes predominam Eichhornia crassipes, Pistia stratiotes, Salvinia spp., entre outras. Estas plantas causam problemas de procriação de mosquitos e de vetores de doenças humanas, impedem atividades de recreação e de pesca e proporcionam condições microaerofílicas, com produção de substâncias de mal odor (Pitelli, 1998).

O controle químico tem se mostrado a opção mais eficaz e viável no controle de plantas aquáticas. Os herbicidas podem ser aplicados às folhas de plantas emergentes e flutuantes ou adicionados à água para controlar plantas submersas (Hance \& Holly, 1990). Cada herbicida possui recomendações específicas para aplicação, que devem ser seguidas para garantir a eficiência e o uso seguro do produto, sem que haja conseqüências à saúde humana e ao ambiente (FAO, 1995).

Atualmente existem cerca de 200 herbicidas (ingredientes ativos) registrados nos Estados Unidos, dos quais seis são registrados para uso aquático. Os herbicidas 2,4-D, diquat, complexos de cobre e endothall têm sido utilizados desde 1940-50; fluridone e glyphosate foram desenvolvidos em 1970; e triclopyr e imazapyr (usados em florestas e auto-estradas) estão sendo avaliados para uso aquático
(Haller, 1998). Por melhor conciliar eficiência e custo, o método de manejo para controle de plantas daninhas aquáticas mais difundido é a utilização dos herbicidas 2,4-D, glyphosate e diquat.

No Brasil, no momento não há nenhum herbicida registrado para controle de aguapé, e a maioria das pesquisas trabalha com herbicidas em condições controladas ou em pequenos corpos hídricos sem fluxo de água; ressaltase que o herbicida fluridone foi registrado em 2002 para controle de plantas imersas, como Egeria spp.

O controle químico promove resultado rápido, sendo uma alternativa de manejo que mantém as plantas daninhas aquáticas abaixo do nível de dano econômico, não prejudicando as funções dos corpos hídricos. Outra vantagem proporcionada pelo controle químico é a especificidade, que elimina o risco da coleta indevida de animais, como ocorre na retirada de plantas dos corpos hídricos utilizando-se o controle mecânico.

O objetivo deste trabalho foi avaliar a sensibilidade de acessos de aguapé, coletados no Estado de São Paulo, a herbicidas.

\section{MATERIAL E MÉTODOS}

Para realização dos testes de controle químico foi feita uma análise da distância genética, considerando cada indivíduo como uma população. A técnica utilizada foi RAPD (Fragmentos de DNA Amplificados ao Acaso). Dentre os vários grupos formados a partir dos dados de identidade e distância genética, foram escolhidos seis materiais de diferentes distâncias, distribuídos ao longo de um dendrograma gerado com dados de variabilidade genética. Selecionaram-se plantas dos seguintes reservatórios: acesso 1 - BAB 05 (Barra Bonita, ponto 05), acesso 2 - JUR 03 (Jurumirim, ponto 03), acesso 3 - EUC 03 (Euclides da Cunha, ponto 03), acesso 4 - PAR 04 (Paraibuna, ponto 04), acesso 5 - TRI 04 (Três Irmãos, ponto 04) e acesso 6 - JUP 02 (Jupiá, ponto 02); cada ponto numerado de acordo com a coleta representou um acesso a ser testado.

Duas plantas de aguapé foram colocadas em caixas de plástico com dimensões de 
$28,0 \times 14,0 \times 12,0 \mathrm{~cm}$, contendo aproximadamente 4 litros de água, que foram alocadas em bancadas de uma casa de vegetação, onde permaneceram crescendo por um período de sete dias, após ser feita uma adubação para garantir a manutenção das plantas durante o teste (Thayer \& Haller, 1985).

A escolha dos herbicidas foi feita considerando produtos para uso em ambientes aquáticos registrados nos Estados Unidos e em fase de testes no Brasil. Foram utilizados os herbicidas imazapyr nas doses de 62,5 e $125,0 \mathrm{~g}$ e.a. ha-1 glyphosate a $1.680 \mathrm{e}$ 3.360 g e.a. ha ${ }^{-1}+0,5 \% \mathrm{~V} / \mathrm{V}$ de Extravon, diquat a 480 e 960 g i.a. ha ${ }^{-1}$ e $2,4-D$ a 670 e $1.340 \mathrm{~g}$ e.a. ha ${ }^{-1}$. O volume de calda utilizado foi de $1931 \mathrm{ha}^{-1}$. A pulverização das plantas foi realizada no laboratório do NUPAM (Núcleo de Pesquisas Avançadas em Matologia). Utilizaram-se bicos de jato plano Teejet $110.02 \mathrm{VS}$ a uma pressão de 1,6 bar. No momento da pulverização a umidade relativa era de $73 \%$ e a temperatura de $25{ }^{\circ} \mathrm{C}$.

O delineamento experimental adotado foi o inteiramente casualizado, com três repetições. A suscetibilidade das plantas aos herbicidas foi avaliada de forma visual. Adotouse uma escala percentual de notas, em que $0 \%$ consistia em nenhum sintoma e $100 \%$ em morte das plantas. As avaliações foram realizadas aos 3, 5, 7, 11, 14, 21 e 28 dias após a aplicação dos diferentes herbicidas.
Os resultados obtidos foram submetidos à análise de variância pelo teste $\mathrm{F}$, e as médias dos tratamentos foram comparadas pelo teste $t$ a $5 \%$ de probabilidade.

\section{RESULTADOS E DISCUSSÃO}

Na Tabela 1 estão apresentados os valores de sensibilidade das plantas de aguapé referentes aos acessos de 1 a 6 em relação ao herbicida imazapyr aplicado nas doses de 62,5 e 125,0 $\mathrm{g} \mathrm{ha}^{-1}$. Em todos os acessos, o herbicida imazapyr causou baixa fitointoxicação inicial. No caso do acesso 2 os sintomas mais severos foram observados a partir dos 14 DAA. Aos 22 DAA, as plantas de aguapé dos seis acessos encontravam-se praticamente mortas, sem condições de rebrota, e aos 28 DAA os seis acessos apresentaram 100\% de controle nas duas doses testadas. O controle proporcionado pelo herbicida imazapyr foi lento e gradual, porém eficiente ao final das avaliações.

Os resultados da eficiência do imazapyr sobre aguapé alcançada neste estudo corroboram os encontrados por Mukhopadhyay \& Bhattacharyya (1985), nos quais o imazapyr a 125,0 $\mathrm{g} \mathrm{ha}^{-1}$ proporcionou morte de plantas de aguapé no período de um mês, quando mantidas em casa de vegetação. De acordo com Martins et al. (1999), o herbicida imazapyr a 125 g e.a. ha ${ }^{-1}$ propiciou $100 \%$ de controle de aguapé aos 28 DAA. As diferenças genéticas encontradas nos acessos testados não estão

Tabela 1 - Sensibilidade de diferentes acessos de Eichhornia crassipes (Mart) Solms ao herbicida imazapyr, em casa de vegetação. Botucatu-SP, 2000

\begin{tabular}{|c|c|c|c|c|c|c|c|c|c|c|c|c|c|c|}
\hline \multirow{4}{*}{ Acesso } & \multicolumn{14}{|c|}{ Porcentagem de controle (dias após aplicação) } \\
\hline & \multicolumn{2}{|c|}{3} & \multicolumn{2}{|r|}{5} & \multicolumn{2}{|c|}{7} & \multicolumn{2}{|c|}{11} & \multicolumn{2}{|c|}{14} & \multicolumn{2}{|c|}{22} & \multicolumn{2}{|c|}{28} \\
\hline & \multicolumn{14}{|c|}{ Dose $\left(\mathrm{g}\right.$ e.a. $\left.\mathrm{ha}^{-1}\right)$} \\
\hline & 62,5 & 125,0 & 62,5 & 125,0 & 62,5 & 125,0 & 62,5 & 125,0 & 62,5 & 125,0 & 62,5 & 125,0 & 62,5 & 125,0 \\
\hline (1) $\mathrm{BAB} 02$ & $1,00 \mathrm{~b}$ & $0,00 \mathrm{c}$ & $1,33 \mathrm{a}$ & $3,00 \mathrm{~b}$ & $2,33 \mathrm{~b}$ & $4,67 \mathrm{~d}$ & $14,00 \mathrm{~cd}$ & $24,33 \mathrm{c}$ & $48,33 \mathrm{ab}$ & $65,33 \mathrm{ab}$ & $98,33 \mathrm{a}$ & $99,33 \mathrm{a}$ & 100,00 & 100,00 \\
\hline (2) JUR 03 & $4,00 \mathrm{a}$ & $7,67 \mathrm{a}$ & $3,00 \mathrm{a}$ & $14,00 \mathrm{a}$ & $7,67 \mathrm{ab}$ & 25,33 a & $25,00 \mathrm{bc}$ & $41,67 \mathrm{a}$ & $49,67 a b$ & $70,33 \mathrm{ab}$ & $98,67 \mathrm{a}$ & $99,63 \mathrm{a}$ & 100,00 & 100,00 \\
\hline (3) EUC 03 & $2,67 \mathrm{ab}$ & $4,00 \mathrm{~b}$ & $2,67 \mathrm{a}$ & $1,33 \mathrm{~b}$ & $4,67 \mathrm{ab}$ & $7,00 \mathrm{~cd}$ & $11,67 \mathrm{~d}$ & $19,33 \mathrm{c}$ & $47,67 \mathrm{ab}$ & $69,33 \mathrm{ab}$ & $99,00 \mathrm{a}$ & $99,67 \mathrm{a}$ & 100,00 & 100,00 \\
\hline (4) PAR 04 & $5,00 \mathrm{a}$ & $5,67 \mathrm{ab}$ & $6,00 \mathrm{a}$ & $10,67 \mathrm{a}$ & $9,00 \mathrm{ab}$ & $17,67 \mathrm{~b}$ & $22,67 \mathrm{bcd}$ & $43,67 \mathrm{a}$ & $67,67 \mathrm{a}$ & $75,67 \mathrm{a}$ & $99,67 \mathrm{a}$ & $99,00 \mathrm{a}$ & 100,00 & 100,00 \\
\hline (5) TRI 04 & $4,00 \mathrm{a}$ & $2,33 \mathrm{bc}$ & $3,67 \mathrm{a}$ & $6,33 \mathrm{ab}$ & $11,00 \mathrm{a}$ & $15,00 \mathrm{~b}$ & 42,67 a & $46,00 \mathrm{a}$ & $67,67 \mathrm{a}$ & $75,00 \mathrm{a}$ & $99,67 \mathrm{a}$ & $100,00 \mathrm{a}$ & 100,00 & 100,00 \\
\hline (6) JUP 02 & $1,00 \mathrm{~b}$ & $3,00 \mathrm{bc}$ & $2,33 \mathrm{a}$ & $5,33 \mathrm{ab}$ & $5,67 \mathrm{ab}$ & $13,00 \mathrm{bc}$ & $26,33 \mathrm{~b}$ & $30,67 \mathrm{~b}$ & $45,67 \mathrm{~b}$ & $57,33 \mathrm{~b}$ & $99,33 \mathrm{a}$ & $99,00 \mathrm{a}$ & 100,00 & 100,00 \\
\hline F trat & $3,54^{* *}$ & $5,36^{* *+}$ & $1,10^{\mathrm{ns}}$ & $2,20^{\text {ns }}$ & $1,85^{\mathrm{ns}}$ & $10,94^{* *}$ & $7,76^{* *}$ & $36,52^{* *-}$ & $2,22^{\text {ns }}$ & $2,58^{\text {ns }}$ & $1,45^{\mathrm{ns}}$ & $0,91^{\mathrm{ns}}$ & - & - \\
\hline $\mathrm{CV}(\%)$ & 52,5 & 52,9 & 82,9 & 82,0 & 59,3 & 28,4 & 28,9 & 9,3 & 21,7 & 0,7 & 0,8 & 0,7 & & \\
\hline d.m.s. & 2,75 & 3,56 & 4,67 & 9,89 & 7,09 & 6,97 & 12,20 & 5,66 & 21,04 & 13,07 & 1,39 & 1,26 & 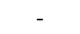 & \\
\hline
\end{tabular}

${ }^{* *}$ significativo a $1 \%$ de probabilidade, ${ }^{*}$ significativo a $5 \%$ de probabilidade e ${ }^{\text {ns }}$ não-significativo.

Médias seguidas de mesma letra, na coluna, não diferem estatisticamente entre si pelo teste $\mathrm{t}(\mathrm{P}>0,05)$. 
provavelmente correlacionadas com algum fator de tolerância ao herbicida imazapyr, que é do grupo das imidazolinonas, as quais apresentam como mecanismo de ação a inibição da enzima acetolactato sintase, impedindo a síntese de aminoácidos de cadeia ramificada, com conseqüente parada de crescimento.

Encontram-se na Tabela 2 os resultados de sensibilidade das plantas de aguapé referente aos acessos em relação ao herbicida diquat aplicado nas doses de 480 e $960 \mathrm{~g} \mathrm{ha}^{-1}$. De modo diferente do herbicida imazapyr, o diquat proporcionou morte rápida de plantas, com mais de $90 \%$ de controle já nos primeiros 3 DAA. Aos 11 DAA, as plantas que não estavam mortas não apresentavam condições de recuperação, como foi o caso dos acessos $2 \mathrm{e}$ 3 tratados com a dose de $480 \mathrm{~g} \mathrm{ha}^{-1}$. O controle das plantas foi eficiente e completo a partir dos 14 DAA. Por ser um herbicida de contato, de ação total, não era esperado que as diferenças genéticas existentes entre os acessos estudados respondessem de forma diferente, uma vez que já se conhecia a eficiência do diquat em plantas de aguapé. Esses resultados reforçaram os encontrados por Mayer (1981), que obteve controle eficiente de plantas de aguapé com a aplicação do herbicida diquat.

Na Tabela 3 estão apresentados os resultados de sensibilidade dos seis acessos testados ao herbicida glyphosate aplicado nas doses de $1.680,0$ e $3.360,0 \mathrm{~g} \mathrm{ha}^{-1}$.

Tabela 2 - Sensibilidade de diferentes acessos de Eichhornia crassipes (Mart) Solms ao herbicida diquat, em casa de vegetação. Botucatu-SP, 2000

\begin{tabular}{|c|c|c|c|c|c|c|c|c|c|c|c|c|c|c|}
\hline \multirow{4}{*}{ Acesso } & \multicolumn{14}{|c|}{ Porcentagem de controle (dias após aplicação) } \\
\hline & \multicolumn{2}{|c|}{3} & \multicolumn{2}{|r|}{5} & \multicolumn{2}{|c|}{7} & \multicolumn{2}{|c|}{11} & \multicolumn{2}{|c|}{14} & \multicolumn{2}{|c|}{22} & \multicolumn{2}{|c|}{28} \\
\hline & \multicolumn{14}{|c|}{ Dose $\left(\mathrm{g}\right.$ e.a. ha $\left.^{-1}\right)$} \\
\hline & 480,0 & 960,0 & 480,0 & 960,0 & 480,0 & 960,0 & 480,0 & 960,0 & 480,0 & 960,0 & 480,0 & 960,0 & 480,0 & 960,0 \\
\hline (1) $\mathrm{BAB} 02$ & $92,67 \mathrm{~b}$ & $94,67 \mathrm{c}$ & $97,00 \mathrm{~b}$ & $97,67 \mathrm{ab}$ & $98,33 \mathrm{bc}$ & $99,00 \mathrm{~b}$ & $100,00 \mathrm{a}$ & 100,00 & 100,00 & 100,00 & 100,00 & 100,00 & 100,00 & 100,00 \\
\hline (2) JUR 03 & $93,00 \mathrm{~b}$ & $95,00 \mathrm{bc}$ & $95,33 \mathrm{c}$ & $96,67 \mathrm{~b}$ & $97,67 \mathrm{c}$ & $99,00 \mathrm{~b}$ & 99,67 ab & 100,00 & 100,00 & 100,00 & 100,00 & 100,00 & 100,00 & 100,00 \\
\hline (3) EUC 03 & $92,67 \mathrm{~b}$ & $96,00 \mathrm{abc}$ & $96,00 \mathrm{bc}$ & $97,67 \mathrm{ab}$ & $97,67 \mathrm{c}$ & $99,00 \mathrm{~b}$ & $99,33 \mathrm{~b}$ & 100,00 & 100,00 & 100,00 & 100,00 & 100,00 & 100,00 & 100,00 \\
\hline (4) PAR 04 & $97,00 \mathrm{a}$ & $97,00 \mathrm{ab}$ & $98,33 \mathrm{a}$ & $98,33 \mathrm{ab}$ & $99,33 \mathrm{ab}$ & $99,33 \mathrm{ab}$ & 100,00 a & 100,00 & 100,00 & 100,00 & 100,00 & 100,00 & 100,00 & 100,00 \\
\hline (5) TRI 04 & $97,00 \mathrm{a}$ & $98,00 \mathrm{a}$ & $98,67 \mathrm{a}$ & $99,00 \mathrm{a}$ & 99,67 a & $100,00 \mathrm{a}$ & 100,00 a & 100,00 & 100,00 & 100,00 & 100,00 & 100,00 & 100,00 & 100,00 \\
\hline (6) JUP 02 & $95,00 \mathrm{ab}$ & $96,00 \mathrm{abc}$ & $98,33 \mathrm{a}$ & $97,67 \mathrm{ab}$ & $99,33 \mathrm{a}$ & $99,33 \mathrm{ab}$ & 100,00 a & 100,00 & 100,00 & 100,00 & 100,00 & 100,00 & 100,00 & 100,00 \\
\hline F trat & $7,33^{* *-1}$ & $3,33^{*}$ & $10,42^{* *}$ & $1,65^{\text {ns }}$ & $7,20^{* *}$ & $1,64^{\mathrm{ns}}$ & $2,10^{\mathrm{ns}}$ & & -- & - - & - & - & - & - \\
\hline $\mathrm{CV}(\%)$ & 1,3 & 1,2 & 0,8 & 1,1 & 0,6 & 0,5 & 0,3 & & - & & & - & - & - \\
\hline d.m.s. & 2,37 & 2,10 & 1,33 & 1,88 & 1,03 & 0,94 & 0,59 & - & - & - & - & - & - & - \\
\hline
\end{tabular}

significativo a $1 \%$ de probabilidade $\mathrm{e}^{\mathrm{ns}}$ não-significativo.

Médias seguidas de mesma letra, na coluna, não diferem estatisticamente entre si pelo teste t $(\mathrm{P}>0,05)$.

Tabela 3 - Sensibilidade de diferentes acessos de Eichhornia crassipes (Mart) Solms ao herbicida glyphosate, em casa de vegetação. Botucatu-SP, 2000

\begin{tabular}{|c|c|c|c|c|c|c|c|c|c|c|c|c|c|c|}
\hline \multirow{4}{*}{ Acesso } & \multicolumn{14}{|c|}{ Porcentagem de controle (dias após aplicação) } \\
\hline & \multicolumn{2}{|c|}{3} & \multicolumn{2}{|c|}{5} & \multicolumn{2}{|r|}{7} & \multicolumn{2}{|c|}{11} & \multicolumn{2}{|c|}{14} & \multicolumn{2}{|c|}{22} & \multicolumn{2}{|c|}{28} \\
\hline & \multicolumn{14}{|c|}{ Dose (g e.a. ha $\left.^{-1}\right)$} \\
\hline & $1.680,0$ & $3.360,0$ & $1.680,0$ & $3.360,0$ & $1.680,0$ & $3.360,0$ & $1.680,0$ & $3.360,0$ & $1.680,0$ & $3.360,0$ & $1.680,0$ & $3.360,0$ & $1.680,0$ & $3.360,0$ \\
\hline (1) $\mathrm{BAB} 02$ & $30,00 \mathrm{~d}$ & $27,00 \mathrm{c}$ & $46,00 \mathrm{~b}$ & $49,00 \mathrm{~d}$ & $67,33 \mathrm{~b}$ & $84,00 \mathrm{c}$ & $87,00 \mathrm{bc}$ & $97,00 \mathrm{ab}$ & $91,67 \mathrm{~b}$ & $99,50 \mathrm{a}$ & $98,33 \mathrm{ab}$ & $100,00 \mathrm{a}$ & $99,67 a b$ & 100,00 \\
\hline (2) JUR 03 & $33,00 \mathrm{~d}$ & $38,00 \mathrm{bc}$ & $50,67 \mathrm{~b}$ & $80,00 \mathrm{~b}$ & $70,67 \mathrm{~b}$ & $85,00 \mathrm{bc}$ & $85,67 \mathrm{c}$ & $98,00 \mathrm{ab}$ & $95,00 \mathrm{ab}$ & $100,00 \mathrm{a}$ & $99,67 \mathrm{a}$ & $100,00 \mathrm{a}$ & $99,67 \mathrm{ab}$ & 100,00 \\
\hline (3) EUC 03 & $45,67 \mathrm{bc}$ & $50,00 \mathrm{ab}$ & $46,67 \mathrm{~b}$ & $51,50 \mathrm{~d}$ & 79,33 a & $77,00 \mathrm{c}$ & $88,67 a b c$ & $93,50 \mathrm{~b}$ & $97,33 \mathrm{a}$ & $98,00 \mathrm{a}$ & $99,33 \mathrm{a}$ & $99,50 \mathrm{a}$ & $99,33 \mathrm{ab}$ & 100,00 \\
\hline (4) PAR 04 & 52,33 a & $55,00 \mathrm{a}$ & $70,67 \mathrm{a}$ & $87,00 \mathrm{a}$ & 81,67 a & $95,00 \mathrm{ab}$ & $91,00 \mathrm{abc}$ & $98,00 \mathrm{ab}$ & $94,33 \mathrm{ab}$ & $100,00 \mathrm{a}$ & $97,00 \mathrm{~b}$ & $100,00 \mathrm{a}$ & $98,67 \mathrm{~b}$ & 100,00 \\
\hline (5) TRI 04 & $47,00 \mathrm{~b}$ & $11,00 \mathrm{~d}$ & $68,33 \mathrm{a}$ & $59,00 \mathrm{c}$ & $80,00 \mathrm{a}$ & $83,00 \mathrm{c}$ & $94,33 \mathrm{a}$ & $98,00 \mathrm{ab}$ & $97,67 \mathrm{a}$ & $99,50 \mathrm{a}$ & $100,00 \mathrm{a}$ & $100,00 \mathrm{a}$ & $100,00 \mathrm{a}$ & 100,00 \\
\hline (6) JUP 02 & $40,67 \mathrm{c}$ & $50,00 \mathrm{ab}$ & $65,00 \mathrm{a}$ & $82,00 \mathrm{~b}$ & 79,67 a & $98,00 \mathrm{a}$ & $93,33 \mathrm{ab}$ & $100,00 \mathrm{a}$ & $97,33 \mathrm{a}$ & $100,00 \mathrm{a}$ & $100,00 \mathrm{a}$ & $100,00 \mathrm{a}$ & $100,00 \mathrm{a}$ & 100,00 \\
\hline F trat & $26,65^{* *}$ & $43,31^{* *}$ & $29,90^{* *}$ & $255,25^{* * *}$ & $4,90^{*}$ & $11,10^{*}$ & $2,28^{\text {ns }}$ & $3,25^{\mathrm{ns}}$ & $1,70^{\mathrm{ns}}$ & $3,00^{\mathrm{ns}}$ & $4,19^{*}$ & $0,47^{\mathrm{ns}}$ & $1,94^{\mathrm{ns}}$ & - \\
\hline $\mathrm{CV}(\%)$ & 7,0 & 9,3 & 6,2 & 1,9 & 6,1 & 3,2 & 4,4 & 1,6 & 3,3 & 0,6 & 1,0 & 0,4 & 0,6 & - \\
\hline d.m.s. & 7,91 & 12,83 & 9,84 & $4,7 \overline{7}$ & 12,69 & 10,56 & 10,93 & 5,95 & 8,57 & 2,25 & 2,74 & 1,59 & 1,71 & - \\
\hline
\end{tabular}

*** significativo a $1 \%$ de probabilidade, ${ }^{*}$ significativo a $5 \%$ de probabilidade e ${ }^{\text {ns }}$ não-significativo.

Médias seguidas de mesma letra, na coluna, não diferem estatisticamente entre si pelo teste t $(\mathrm{P}>0,05)$. 
Pode-se observar para o acesso 5, aos 3 DAA, que o glyphosate aplicado na dose de 3.360,0 $\mathrm{g} \mathrm{ha}^{-1}$ apresentou fitointoxicação de apenas $11 \%$. Esse fato não interferiu na obtenção de $100 \%$ de controle aos 22 DAA, equiparando-se aos demais acessos.

Este estudo está de acordo com os resultados alcançados por Gelmini (1996), relativos à sensibilidade do glyphosate aplicado na dose de $3,89 \mathrm{~kg} \mathrm{ha}^{-1}$, que, aos $14 \mathrm{DAA}$, proporcionou 90\% de controle em plantas de aguapé, além de corroborar os resultados de Iamauti \& Victoria Filho (1998), que comprovaram a eficiência do herbicida glyphosate para essa espécie.

No entanto, segundo Shinohara et al. (1988), a menor dose de glyphosate que apresentou resultados satisfatórios foi de $3.240 \mathrm{~g} \mathrm{ha}^{-1}$, divergindo da dose de $1.680,0 \mathrm{~g}$ e.a. ha- ${ }^{-1}$, que apresentou $98 \%$ de controle das plantas aos 28 DAA. Ficou evidente que existem diferenças em alguns materiais quando da aplicação da meia dose, sendo os acessos 1 a 4 menos sensiveis ao glyphosate, o que pode ser contornado com a aplicação da dose cheia ou trazer preocupações quanto à aplicação de subdoses. Deve-se ressaltar que em campo provavelmente a eficiência fosse menor, como observado por Martins et al. (1999), os quais, também em condições controladas, porém com as caixas d'água em campo, constataram controle bem inferior ao ora observado com a aplicação do herbicida glyphosate a $3.360,0 \mathrm{~g} \mathrm{ha}^{-1}$, que proporcionou controle em torno de $83 \%$, aos 28 DAA.
Na Tabela 4 estão apresentados os resultados de sensibilidade de diferentes acessos ao herbicida 2,4-D aplicado nas doses de 670,0 e $1.340,0 \mathrm{~g} \mathrm{ha}^{-1}$. Conforme observado por Gupta \& Subbaiah (1982), Rao \& Subrahmanyan (1987) e Surjit-Singh et al. (1993), os resultados ora observados, sobre o efeito do herbicida 2,4-D, comprovaram sua eficiência no controle de plantas de aguapé. Independentemente do fato de que aos 28 DAA todos os acessos apresentaram um controle total de plantas para as duas doses de 2,4-D testadas, nota-se que o acesso 6 respondeu de maneira diferenciada quando da aplicação da menor dose de 2,4-D, dependendo do período analisado, e, de modo geral, em quase todas as épocas de avaliação apresentou menor sensibilidade ao 2,4-D. Esses resultados confirmam os de Martins et al. (2002). Em um outro estudo, Neves et al. (2002) também obtiveram $100 \%$ de controle em plantas de aguapé aos 20 DAA com doses de 1.340 e $2.680 \mathrm{~g} \mathrm{ha}^{-1}$. Apesar de a dose de $2.680 \mathrm{~g} \mathrm{ha}^{-1}$ propiciar controle total das plantas aos 20 DAA, deve-se considerar a utilização da menor dose, por proporcionar economia de produto e eficiência comprovada no controle de plantas aquáticas.

As variabilidades genéticas existentes entre os acessos de aguapé aparentemente não se refletiram em alterações nas plantas que proporcionassem respostas diferenciadas aos herbicidas testados, visto que ao final do período de estudo todos os tratamentos utilizados

Tabela 4 - Sensibilidade de diferentes acessos de Eichhornia crassipes (Mart) Solms ao herbicida 2,4-D, em casa de vegetação. Botucatu-SP, 2000

\begin{tabular}{|c|c|c|c|c|c|c|c|c|c|c|c|c|c|c|}
\hline \multirow{4}{*}{ Acesso } & \multicolumn{14}{|c|}{ Porcentagem de controle (Dias após aplicação) } \\
\hline & \multicolumn{2}{|c|}{3} & \multicolumn{2}{|c|}{5} & \multicolumn{2}{|c|}{7} & \multicolumn{2}{|c|}{11} & \multicolumn{2}{|c|}{14} & \multicolumn{2}{|c|}{22} & \multicolumn{2}{|c|}{28} \\
\hline & \multicolumn{14}{|c|}{ Dose (g e.a. ha $\left.{ }^{-1}\right)$} \\
\hline & 670,0 & $1.340,0$ & 670,0 & $1.340,0$ & 670,0 & $1.340,0$ & 670,0 & $1.340,0$ & 670,0 & $1.340,0$ & 670,0 & $1.340,0$ & 670,0 & $1.340,0$ \\
\hline (1) $\mathrm{BAB} 02$ & $48,33 \mathrm{bc}$ & $52,33 \mathrm{bc}$ & $68,00 \mathrm{a}$ & $69,00 \mathrm{a}$ & $70,67 \mathrm{ab}$ & $80,33 \mathrm{a}$ & 88,67 a & 92,67 a & $95,00 \mathrm{a}$ & $99,33 \mathrm{a}$ & $99,33 \mathrm{a}$ & $100,00 \mathrm{a}$ & 100,00 & 100,00 \\
\hline (2) JUR 03 & $45,33 \mathrm{c}$ & $56,67 \mathrm{~b}$ & $46,00 \mathrm{e}$ & $74,33 \mathrm{a}$ & $56,67 \mathrm{c}$ & $85,33 \mathrm{a}$ & $73,33 \mathrm{~b}$ & $92,33 \mathrm{a}$ & $84,00 \mathrm{c}$ & $96,33 \mathrm{a}$ & $99,00 \mathrm{a}$ & $100,00 \mathrm{a}$ & 100,00 & 100,00 \\
\hline (3) EUC 03 & $60,00 \mathrm{a}$ & $68,67 \mathrm{a}$ & $49,67 \mathrm{de}$ & $70,67 \mathrm{a}$ & $71,00 \mathrm{ab}$ & $85,00 \mathrm{a}$ & 85,67 a & $94,00 \mathrm{a}$ & $90,67 \mathrm{ab}$ & $98,00 \mathrm{a}$ & $99,67 \mathrm{a}$ & $100,00 \mathrm{a}$ & 100,00 & 100,00 \\
\hline (4) PAR 04 & $52,00 \mathrm{~b}$ & $50,67 \mathrm{bc}$ & $62,33 \mathrm{~b}$ & 64,33 a & $74,33 \mathrm{a}$ & $74,00 \mathrm{a}$ & $91,00 \mathrm{a}$ & 89,33 a & 94,67 a & $94,00 \mathrm{a}$ & 99,67 a & $98,00 \mathrm{~b}$ & 100,00 & 100,00 \\
\hline (5) TRI 04 & $48,00 \mathrm{bc}$ & $51,33 \mathrm{bc}$ & $55,67 \mathrm{c}$ & $64,00 \mathrm{a}$ & $66,67 \mathrm{~b}$ & $74,33 \mathrm{a}$ & 88,00 a & $95,00 \mathrm{a}$ & $92,00 \mathrm{ab}$ & $97,33 \mathrm{a}$ & 98,67 a & $100,00 \mathrm{a}$ & 100,00 & 100,00 \\
\hline (6) JUP 02 & $39,33 \mathrm{~d}$ & $47,33 \mathrm{c}$ & $52,00 \mathrm{~cd}$ & 57,67 a & $59,00 \mathrm{c}$ & $70,00 \mathrm{a}$ & 85,33 a & 88,67 a & $90,00 \mathrm{~b}$ & $93,00 \mathrm{a}$ & 99,67 a & $100,00 \mathrm{a}$ & 100,00 & 100,00 \\
\hline F trat & $25,97^{* *}$ & $8,66^{*}$ & $39,42^{* *}$ & $1,19^{\text {ns }}$ & $16,69^{* *}$ & $0,82^{\text {ns }}$ & $9,29^{* *}$ & $0,49^{\text {ns }}$ & $7,31^{*}$ & $1,19^{\mathrm{ns}}$ & $1,20^{\mathrm{ns}}$ & $3,00^{\mathrm{ns}}$ & - & - \\
\hline $\mathrm{CV}(\%)$ & 4,8 & 8,2 & 4,1 & 14,1 & 4,5 & 15,6 & 4,2 & 6,8 & 2,8 & 4,0 & 0,7 & 0,8 & - & - \\
\hline d.m.s. & 4,17 & 7,92 & 4,04 & 16,67 & 5,35 & 21,63 & 6,30 & 11,07 & 4,57 & 6,85 & 1,19 & 1,45 & - & - \\
\hline
\end{tabular}

*** significativo a $1 \%$ de probabilidade $\mathrm{e}^{\mathrm{ns}}$ não-significativo.

Médias seguidas de mesma letra, na coluna, não diferem estatisticamente entre si pelo teste t $(\mathrm{P}>0,05)$. 
proporcionaram 100\% de controle. As diferenças notadas em relação aos sintomas de fitointoxicação foram dependentes do herbicida avaliado.

O diquat foi o herbicida que provocou maior fitointoxicação inicial, com queima generalizada da planta aos 3 DAA. Esses dados reforçaram os obtidos por Alimi \& Akinnyemiju (1990), que avaliaram a eficiência de diquat nas doses de 2.000 e $4.000 \mathrm{~g} \mathrm{ha}^{-1}$ para controlar plantas de aguapé na Nigéria. A ação rápida deste herbicida pode se tornar inconveniente, por causa da intensa depleção dos teores de oxigênio dissolvido na água (Neves et al., 2002). O manejo das plantas em pequenos corpos hídricos com grande infestação deve ser planejado de forma a evitar a decomposição de grande massa vegetal em curto espaço de tempo. Apesar de ser um método mais dispendioso que o uso de outros herbicidas, como o 2,4-D, a utilização do diquat deve ser considerada, por promover controle rápido e seguro, com restrição máxima de 5 DAA para o uso da água na irrigação de culturas.

Dos herbicidas testados, apenas o glyphosate na dose de $1.680,0 \mathrm{~g} \mathrm{ha}^{-1}$ não proporcionou $100 \%$ de controle das plantas de aguapé aos 28 DAA, sendo, porém, perfeitamente recomendável. Considerando que esses testes foram conduzidos em casa de vegetação, os resultados obtidos concordam com os alcançados por Gutierrez-Lopez (1993), que, ao testar glyphosate nas doses de $2.380,0$ e $3.360 \mathrm{~g} \mathrm{ha}^{-1}$ em diferentes densidades de aguapé, obteve, com ambas as doses, $100 \%$ de controle das plantas de aguapé sob condições de baixa densidade $\left(10 \mathrm{~kg} \mathrm{~m}^{-2}\right)$, aos 33 DAA.

Thayer \& Haller (1985), ao avaliarem os efeitos dos herbicidas diquat a $1.100 \mathrm{~g} \mathrm{ha}^{-1} \mathrm{e}$ glyphosate a $1.500,0$ e 6.700,0 $\mathrm{g} \mathrm{ha}^{-1} \mathrm{em}$ alfaced'água (Pistia stratiores), observaram aos 15 DAA $100 \%$ de controle com diquat e 96 e 99\% de controle com glyphosate nas duas doses testadas, respectivamente. Pelo fato de a alface-d'água ser também uma planta daninha aquática flutuante livre, assim como o aguapé, a eficiência de controle obtida com esses herbicidas pode facilitar a resolução dos problemas oriundos da infestação de áreas que possuem as duas plantas convivendo.

Depois do diquat, o herbicida 2,4-D foi o que causou maior fitointoxicação inicial, porém com morte das plantas no dobro do período (28 DAA) em relação ao diquat. Por ser uma auxina sintética, o 2,4-D provoca desordem na divisão celular, que pode ser observada pelo crescimento irregular das partes da planta e retorcimento dos bordos foliares, com posterior amarelecimento.

Em termos gerais, pode-se observar que as diferenças verificadas entre os acessos durante a fase intermediária do estudo foram anuladas ao final do período de testes, em que todos os materiais analisados apresentaram fitointoxicações semelhantes, com o controle total das plantas de aguapé.

Durante as décadas de 70 e 80 , vários estudos sobre o controle químico de aguapé foram realizados. Atualmente, ponderando os danos causados por essa planta daninha aquática, poucos estudos estão sendo realizados, talvez pelo fato de não terem sido introduzidos novos herbicidas para ambiente aquático. $\mathrm{O}$ Brasil encontra-se em situação inferior ao ser comparado a países desenvolvidos, como EUA, Austrália e Nova Zelândia. No entanto, cada vez mais esforços estão sendo direcionados para o conhecimento do efeito de herbicidas sobre as plantas daninhas aquáticas nativas e exóticas existentes no País. Talvez, em pouco tempo isso possibilite o entendimento mais apurado da eficiência dos herbicidas sobre as comunidades de plantas daninhas aquáticas e da sua dinâmica no meio.

\section{LITERATURA CITADA}

ALIMI, T.; AKINNYEMIJU, A. An economic analysis of water hyacinth control methods in Nigeria. J. Aquatic Plant Manag., v. 28, p. 105-107, 1990.

BITAR, A. L.; SIKAR, B. M. Taxa de crescimento e produção primária em duas espécies de macrófitas aquáticas (Eichhornia crassipes e Pistia stratiotes) no reservatório de Salto Grande-SP. In: CONGRESSO BRASILEIRO DE LIMNOLOGIA, 7., 1999, FLorianópolis. Resumos...

Florianópolis: 1999. p. 72.

FAO. Strategies for water hyacinth control. Report of a Panel of Experts Meeting. Flórida, 1995. p. 137-148.

GELMINI, G. A. Controle químico de Aguapé (Eichhornia crassipes Mart. Solms) e de alface d'água (Pistia stratiotes L). 1996. 205 f. Dissertação (Mestrado em Fitotecnia) - Escola Superior de Agricultura "Luiz de Queiroz", Piracicaba, 1996. 
GUPTA, K. M.; SUBBAIAH, G. Chemical control of water hyacinth. In: ANNUAL CONFERENCE OF INDIAN SOCIETY OF WEED SCIENCE, 1982. Abstracts of papers... s.l.: s. n., 1982.

GUTIERREZ-LOPEZ, E. Effect of glyphosate on different densities of water hyacinth. J. Aquatic Plant Manag., v. 31, p. 255-257, 1993.

IAMAUTI, M. T.; VICTORIA FILHO, R. Efeito da adição de surfactante no controle do aguapé (Eichhornia crassipes) com glyphosate. In: CONGRESSO BRASILEIRO DE HERBICIDAS E PLANTAS DANINHAS, 8.,1998, Piracicaba. Resumos... Piracicaba: 1998. p. 348.

HALLER, W. T. Options for mechanical and chemical aquatic weed control. In: WORKSHOP CONTROLE DE PLANTAS AQUÁTICAS, 1998, Brasília. Resumos... Brasília: 1998. p. 46-53.

MARTINS, D. et al. Controle químico de plantas daninhas aquáticas em condições controladas - caixas d'água. Planta Daninha, v. 17, n. 2, p. 289-296, 1999.

MARTINS, D. et al. Controle químico de Pistia stratiotes, Eichhornia crassipes e Salvínia molesta em caixas d' água. Planta Daninha, v. 20, p. 83-88, 2002.

MAYER, H. G. Chemical control of water hyacinth (Eichhornia crassipes Solms) considering least enviromental disturbance. Acta Hydrochim. Hydrobiol., v. 9, n. 1, p. 57-68, 1981.
MUKHOPADHYAY, S. K.; BHATTACHARYYA, M. Evalution of new herbicide AC 252925 (arsenal) in controlling water hyacinth and Parthenium weeds. In: ANNUAL CONFERENCE OF INDIAN SOCIETY OF WEED SCIENCE, 1985. Abstracts of papers... s.l.: s.n., 1985.

NEVES, T.; FOLONI, L. L.; PITELLI, R. A. Controle químico do aguapé (Eichhornia crassipes). Planta Daninha, v. 20, p. 88-97, 2002.

PITELLI, R. A. Macrófitas Aquáticas no Brasil, na condição de problemáticas. In: WORKSHOP CONTROLE DE PLANTAS AQUÁTICAS, 1998, Brasília. Resumos... Brasília: 1998. p. 12-15.

RAO, A. S.; SUBRAHMANYAM, K. Chemical control of water hyacinth (Eichhornia crassipes (Mart) Solms). Ind. J. Agron., v. 32, n. 2, p. 182-183, 1987.

SHINOHARA, R. K. et al. Controle de aguapé (Eichhornia crassipes) e alface-d'água (Pistia stratiotes) com a utilização do glyphosate. In: CONGRESSO BRASILEIRO DE HERBICIDAS E PLANTAS DANINHAS, 18., 1998, Piracicaba. Resumos... Piracicaba: 1998. p. 346-347.

SURJIT-SINGH, VATS, O. P.; SINGH, S. Integrated weed management for sustainable agriculture. In: INDIAN SOCIETY OF WEED SCIENCE INTERNATIONAL SYMPOSIUM. Proceedings... Hisar, 1993. p. 244-246.

THAYER, D. D.; HALLER, W. T. Effect of herbicides on floating aquatic plants. J. Aquatic Plant Manag., v. 23, p. 94-95, 1985. 